\title{
ASCENT, DESCENT AND COMPACT PERTURBATIONS
}

\author{
SANDY GRABINER
}

\begin{abstract}
The collections of upper semi-Fredholm operators with finite ascent and of lower semi-Fredholm operators with finite descent are both closed under commuting compact perturbations.
\end{abstract}

Suppose that $T$ and $V$ are commuting bounded linear operators on the Banach space $X$ and that $T-V$ is compact. In Theorem 2 below we show that if $T$ is upper semi-Fredholm, then $T$ has finite ascent if and only if $V$ does; and, dually, if $T$ is lower semi-Fredholm, it has finite descent if and only if $V$ has finite descent. If $T$ is invertible or just Fredholm of index 0 , it has long been known that $V$ has finite ascent and descent [3, Theorem 6.3, p. 610], [1, Theorem (1.4.5), p. 12], [2, pp. 39-42]; but, even in this special case, examples show that the commutativity of $T$ and $V$ is crucial [3, p. 599], [1, pp. 13-14], [2, p. 40].

We start with a lemma which treats the special case that $T$ is onto.

LEMMA 1. Suppose that $T$ and $V$ are commuting bounded linear operators on the Banach space $X$. If $T-V$ is compact and $T$ is onto, then $V$ has finite descent.

Proof. For each nonnegative integer $k$, the range, $R\left(V^{k}\right)$, has finite codimension [1, Corollary (1.3.7)(b), p. 9] and the map induced by $T$ on $X / R\left(V^{k}\right)$ is onto. Therefore this induced map is one-to-one, so that the null-space $N(T) \subseteq R\left(V^{k}\right)$. Since $T$ is onto, there is a positive number $\gamma$ for which $\|T x\| \geqslant \gamma \operatorname{dist}(x, N(T))$ for all $x$ in $X$. Suppose that $x$ belongs to $X$ and $z$ belongs to $R\left(V^{k}\right)$; then $T\left(R\left(V^{k}\right)\right)=R\left(V^{k} T\right)=R\left(V^{k}\right)$ so there is a $y$ in $R\left(V^{k}\right)$ with $T y=z$. Thus we have $\|T x-z\|=\|T(x-y)\| \geqslant \gamma \operatorname{dist}(x-$ $y, N(T)) \geqslant \gamma \operatorname{dist}\left(x, R\left(V^{k}\right)\right)$, since $N(T) \subseteq R\left(V^{k}\right)$. Since this holds for all $z$ in $R\left(V^{k}\right)$, we obtain

$$
\operatorname{dist}\left(T x, R\left(V^{k}\right)\right) \geqslant \gamma \operatorname{dist}\left(x, R\left(V^{k}\right)\right) .
$$

Suppose $V$ had infinite descent. Then there would be a bounded sequence $\left\{x_{n}\right\}$ with $x_{n} \in R\left(V^{n}\right)$ and $\operatorname{dist}\left(x_{n}, R\left(V^{n+1}\right)\right) \geqslant 1$. Let $K=T-V$ and suppose $m>n$. Then $K x_{m}-K x_{n}=\left(K x_{m}+(T-K) x_{n}\right)-T x_{n}$. So that

$$
\left\|K x_{m}-K x_{n}\right\| \geqslant \operatorname{dist}\left(T x_{n}, R\left(V^{n+1}\right)\right) \geqslant \gamma \operatorname{dist}\left(x_{n}, R\left(V^{n+1}\right)\right) \geqslant \gamma .
$$

Received by the editors July 19, 1977 and, in revised form, January 24, 1978.

AMS (MOS) subject classifications (1970). Primary 47A55, 47B05; Secondary 47B30. 
But this contradicts the compactness of $K$, so $V$ must have finite descent.

THEOREM 2. Suppose that $T$ and $V$ are commuting bounded linear operators on the Banach space $X$ with $T-V$ compact.

(A) If $T$ is upper semi-Fredholm, then $V$ has finite ascent if and only if $T$ has finite ascent.

(B) If $T$ is lower semi-Fredholm, then $V$ has finite descent if and only if $T$ has finite descent.

Proof. Suppose first that $T$ is lower semi-Fredholm. Since $V$ is also lower semi-Fredholm [1, Corollary (1.3.7)(b), p. 9], it will be enough to show that $V$ has finite descent if $T$ has. Let $p$ be an integer with $R\left(T^{p}\right)=R\left(T^{p+1}\right)$. Then $R\left(T^{p}\right)$ is a closed subspace of finite codimension [1, Corollary (1.3.3), p. 9] and the restriction of $T$ to $R\left(T^{p}\right)$ is onto. Therefore, by Lemma 1, the restriction of $V$ to $R\left(T^{p}\right)$ has finite descent, so that there is an integer $k$ for which

$$
R\left(V^{m}\right) \supseteq R\left(V^{m} T^{p}\right)=R\left(V^{k} T^{p}\right)
$$

for all $m \geqslant k$. Since $R\left(V^{k} T^{p}\right)$ has finite codimension [1, Corollary (1.3.3), p. 9], $V$ has finite descent. This proves (B).

Now suppose that $T$ and $V$ are upper semi-Fredholm. Then $T^{*}$ and $V^{*}$ are lower semi-Fredholm, and the ascent of $T$ and $V$, respectively, equals the descent of $T^{*}$ and of $V^{*}$, respectively [1, pp. 7-8]. Part (A) now follows directly from Part (B).

Instead of proving Part (A) from Part (B) in Theorem 2 by duality arguments, we could have proved the dual result to Lemma 1 for $T$ bounded below and then used this result to prove Part (A). The direct proofs are very similar to our proofs of Lemma 1 and Theorem 2(B).

In subsequent papers we will use the results of the present paper to study compact perturbations of more general classes of operators.

\section{REFERENCES}

1. S. R. Caradus, W. E. Pfaffenberger and B. Yood, Calkin algebras and algebras of operators on Banach spaces, Lecture Notes in Pure and Appl. Math., vol. 9, Dekker, New York, 1974.

2. M. A. Kaashoek and D. C. Lay, Ascent, descent, and commuting perturbations, Trans. Amer. Math. Soc. 189 (1972), 35-47.

3. B. Yood, Properties of linear transformations preserved under addition of a completely continuous transformation, Duke Math. J. 18 (1951), 599-612.

Department of Mathematics, Pomona College, Claremont, California 91711 